\title{
COMPARAÇÃO DE TÉCNICAS PARA O CÁLCULO DE IDEMPOTENTES GERADORES DE CÓDIGOS CÍCLICOS
}

\author{
Gustavo Terra Bastos, Marinês Guerreiro, \\ Departamento de Matemática - DMA, UFV, \\ 36.570-000, Viçosa, MG \\ E-mail: gtbastos@yahoo.com.br, marines@ufv.br/
}

\begin{abstract}
Resumo: Neste trabalho apresentamos técnicas recentes para o cálculo de idempotentes primitivos em álgebras de grupo ou em álgebras polinomiais. Esses idempotentes podem ser vistos como geradores de códigos cíclicos minimais. Exibiremos duas abordagens para tais cálculos: a primeira, que chamaremos abordagem polinomial, é realizada no anel das classes residuais módulo $x^{n}-1$ de um anel de polinômios, onde $n$ denota o comprimento do código. Já a segunda é realizada no contexto de álgebras de grupo de grupos abelianos sobre corpos finitos de ordem prima. Em particular, consideramos grupos cíclicos de ordem $n$ e apresentamos um isomorfismo entre o anel de classes residuais e a álgebra de grupo de modo que possamos trabalhar livremente nestas duas abordagens.
\end{abstract}

Palavras-chave: Códigos cíclicos minimais, Idempotentes primitivos, $\lambda$-aplicação

\section{Códigos Cíclicos}

Seja $n \geq 1$ um número natural e $\mathbb{F}_{l}$ um corpo finito com $l$ elementos. Um código linear de comprimento $n$ é um subespaço $\mathfrak{C} \subset \mathbb{F}_{l}^{n}$ (inclusão própria).

Os códigos cíclicos são um caso particular de códigos lineares. Nesta seção apresentamos os códigos cíclicos como ideais de um anel de polinômios e ideais em uma álgebra de grupo para um grupo cíclico sobre um corpo finito. Estas duas abordagens serão usadas durante todo o trabalho.

Definimos a permutação cíclica de coordenadas em $\mathbb{F}_{l}^{n}$, por

$$
\begin{aligned}
& T_{\pi}: \quad \mathbb{F}_{l}^{n} \quad \rightarrow \quad \mathbb{F}_{l}^{n} \\
& c=\left(c_{0}, \ldots, c_{n-1}\right) \mapsto T_{\pi}(c)=\left(c_{n-1}, c_{0}, \ldots, c_{n-2}\right) .
\end{aligned}
$$

Definição 1.1. Um código linear $\mathfrak{C}$ é chamado código cíclico se, para toda palavra $u=\left(u_{0}, u_{1}, \ldots, u_{n-1}\right)$ em $\mathfrak{C}$, o vetor $T_{\pi}(u)=\left(u_{n-1}, u_{0}, \ldots, u_{n-2}\right)$ também está em $\mathfrak{C}$, ou seja, $T_{\pi}(\mathfrak{C}) \subset \mathfrak{C}$

Considere $\mathcal{R}_{n}$ o anel das classes residuais em $\mathbb{F}_{l}[X]$ módulo $X^{n}-1$, ou seja,

$$
\mathcal{R}_{n}=\frac{\mathbb{F}_{l}[X]}{\left\langle X^{n}-1\right\rangle} .
$$

Todo elemento de $\mathcal{R}_{n}$ pode ser representado de forma única por um polinômio

$$
a_{0}+a_{1} X+\cdots+a_{n-1} X^{n-1}
$$

de grau no máximo $n-1$. 
Os códigos cíclicos podem ser realizados de diferentes formas. É fácil verificar que

$$
\begin{aligned}
\varphi: \quad \mathbb{F}_{l}^{n} & \longrightarrow \mathcal{R}_{n} \\
\left(b_{0}, \ldots, b_{n-1}\right) & \mapsto \sum_{i=0}^{n-1} b_{i} X^{i}
\end{aligned}
$$

é um isomorfismo e que os códigos cíclicos em $\mathbb{F}_{l}^{n}$ correspondem aos ideais no anel quociente $\mathcal{R}_{n}$.

Podemos também considerar os códigos cíclicos como ideais na álgebra de grupo de um grupo cíclico. De fato, dado $G=\left\langle a / a^{n}=1\right\rangle$ um grupo cíclico finito de ordem $n$, definimos a aplicação

$$
\begin{aligned}
\psi: \quad \mathbb{F}_{l}^{n} & \longrightarrow \mathbb{F}_{l} G \\
\left(b_{0}, \ldots, b_{n-1}\right) & \mapsto \sum_{i=0}^{n-1} b_{i} a^{i} .
\end{aligned}
$$

É fácil verificar que $\psi$ é um isomorfismo de $\mathbb{F}_{l}$-espaços vetoriais.

Teorema 1.2. Um subespaço vetorial $\mathfrak{C} d e \mathbb{F}_{l}^{n}$ é um código cíclico se, e somente se, $\psi(\mathfrak{C})$ é um ideal de $\mathbb{F}_{l} G$.

Por (2) e (3), temos o isomorfismo $\psi \varphi^{-1}$ entre $\mathcal{R}_{n}$ e $\mathbb{F}_{l} G$ tal que

$$
\begin{array}{ccccc} 
& \varphi^{-1} & & \psi & \\
\mathcal{R}_{n} & \longrightarrow & \mathbb{F}_{l}^{n} & \longrightarrow & \mathbb{F}_{l} G \\
\sum_{i=0}^{n-1} b_{i} X^{i} & \mapsto & \left(b_{0}, b_{1}, \ldots, b_{n-1}\right) & \mapsto & \sum_{i=0}^{n-1} b_{i} a^{i} .
\end{array}
$$

Verifica-se facilmente que este é um isomorfismo de álgebras.

De posse dessas informações, apresentamos as técnicas para o cálculo de idempotentes primitivos em ambos os contextos, a saber, o polinomial e o de álgebra de grupo. Dado o isomorfismo (4), podemos trabalhar livremente em ambas as abordagens, considerando que na segunda (álgebra de grupo) o grupo seja cíclico.

Ficará claro durante a exibição das técnicas quais são as vantagens e desvantagens de cada uma.

\section{Primeira abordagem - polinomial}

\section{$2.1 \lambda$-Aplicação e Idempotentes Primitivos em Anéis Semissimples}

Vamos expor neste capítulo um método para obter códigos cíclicos minimais a partir de uma abordagem polinomial. Chamamos este método de $\lambda$-técnica e ela é definida a partir de uma $\lambda$-aplicação e um $\lambda$-produto de polinômios, que são definidos a seguir. A principal referência para esta seção é [6].

\subsection{A $\lambda$-aplicação e as classes ciclotômicas}

Nesta seção consideramos a seguinte hipótese geral:

$$
\begin{aligned}
& r \geqslant 2 \text { e } \alpha_{i} \geqslant 1 \text { são números inteiros, } \\
& m=\prod_{i=1}^{r} p_{i}^{\alpha_{i}}, \text { com } p_{i} \text { primos ímpares distintos, para } 1 \leq i \leq r, \\
& m d c\left(\frac{\varphi\left(p_{i}^{\alpha_{i}}\right)}{2}, \frac{\varphi\left(p_{j}^{\alpha_{j}}\right)}{2}\right)=1, \text { para } 1 \leq i \neq j \leq r, \\
& l \neq 2 \text { é uma raiz primitiva } \bmod p_{i}^{\alpha_{i}}, \text { para cada } 1 \leq i \leq r \text { e } \operatorname{mdc}(l, m)=1,
\end{aligned}
$$


Considere o conjunto $A=\{0,1, \ldots,(m-1)\}$. Para $a, b \in A$, definimos

$$
a \sim b \text { se, e somente se, } a \equiv b l^{i}(\bmod m), \text { para algum inteiro } i \geq 0 .
$$

Esta é uma relação de equivalência no conjunto $A$ cujas classes de equivalência são chamadas classes $l$-ciclotômicas de $A$.

Se $t$ é o menor inteiro positivo tal que $s \equiv s l^{t}(\bmod m)$, então a classe $l$-ciclotômica de $s$ é definida por $C_{s}=\left\{s, s l, \ldots, s l^{t-1}\right\}$.

Notações 2.1. Sob a hipótese geral (5), estabelecemos as seguintes notações e definições:

(i) Para $1 \leq i \leq r, \bar{m}_{i}=\frac{m}{p_{i}^{\alpha_{i}}}$.

(ii) $A_{i}=\left\{0,1,2, \ldots, p_{i}^{\alpha_{i}}-1\right\}$ e $A=\{0,1, \ldots,(m-1)\}$.

(iii) $R_{i}$ e $N_{i}$ são os conjuntos de resíduos quadráticos e não quadráticos módulo $p_{i}^{\alpha_{i}}$.

(iv) $R_{p_{i}^{\beta_{i}}}=\left\{r p_{i}^{\beta_{i}} / r \in R_{i}\right\}, N_{p_{i}^{\beta_{i}}}=\left\{s p_{i}^{\beta_{i}} / s \in N_{i}\right\}, X_{i}=R_{i}\left(\right.$ ou $\left.N_{i}\right)$ e $Y_{i}=N_{i}\left(\right.$ ou $\left.R_{i}\right)$.

(v) $\mathcal{R}_{m}=\frac{\mathbb{F}_{l}[x]}{\left\langle x^{m}-1\right\rangle}$ e $\mathcal{R}_{p_{i}^{\alpha_{i}}}=\frac{\mathbb{F}_{l^{2}}[x]}{\left\langle x^{\left.p_{i}^{\alpha_{i}}-1\right\rangle}\right.}$.

(vi) $\delta$ é uma raiz m-ésima primitiva da unidade em alguma extensão do corpo $\mathbb{F}_{l^{2}}$.

(vii) Para qualquer $m$, sempre denotamos a classe ciclotômica $C_{0}$ módulo $m$ por $\{0\}$.

Definição 2.2 ([6], Definition 2.2). Dado o produto cartesiano $A_{1} \times A_{2} \times \ldots \times A_{r}$, definimos a $\lambda$-aplicação por

$$
\begin{aligned}
\lambda: A_{1} \times A_{2} \times \ldots \times A_{r} & \longrightarrow A_{r}^{A} \\
\left(a_{1}, \ldots, a_{r}\right) & \mapsto \sum_{k=1}^{r} a_{k} \bar{m}_{k}(\bmod m),
\end{aligned}
$$

O seguinte resultado é o conhecido Teorema Chinês do Resto.

Lema 2.3. A $\lambda$-aplicação é uma bijeção. (A $\lambda$-aplicação é um isomorfismo de grupos abelianos.)

Observação 2.4 ([6], Theorem 2.4). Sob a hipótese geral (5), $\frac{\varphi\left(\prod_{i=1}^{r} p_{i}^{\beta_{i}}\right)}{2^{r-1}}$ é a ordem de l módulo $\prod_{i=1}^{r} p_{i}^{\beta_{i}}$, para $1 \leq \beta_{i} \leq \alpha_{i}$. Ressaltamos que esse resultado é um caso particular da função de Carmichael.

Observação 2.5. De [6], a $\lambda$-aplicação faz corresponder a cada classe l-ciclotômica de A a união de dois produtos cartesianos de classes $l^{2}$-ciclotômicas módulo $p_{i}^{\alpha_{i}}$, para cada $1 \leq i \leq r$, a saber, $X_{p_{1}^{\beta_{1}}} \times \ldots \times X_{p_{r}^{\beta_{r}}} \cup Y_{p_{1}^{\beta_{1}}} \times \ldots \times Y_{p_{r}^{\beta_{r}}}$ e vice-versa, onde $X_{p_{i}^{\beta_{i}}}=R_{p_{i}^{\beta_{i}}}\left(\right.$ ou $\left.N_{p_{i}^{\beta_{i}}}\right) e$ $Y_{p_{i}^{\beta_{i}}}=N_{p_{i}^{\beta_{i}}}\left(\right.$ ou $\left.R_{p_{i}^{\beta_{i}}}\right)$

Lema 2.6. Sob a hipótese geral (5), para cada $1 \leq i \leq r$ e $1 \leq \beta_{i} \leq \alpha_{i}-1$, os conjuntos $R_{i}, N_{i}, R_{p_{i}^{\beta_{i}}}$ e $N_{p_{i}^{\beta_{i}}}$ são as classes $l^{2}$-ciclotômicas módulo $p_{i}^{\alpha_{i}}$.

Teorema 2.7. Sob a hipótese geral (5), o número de classes l-ciclotômicas módulo m é

$$
\frac{\left(2 \alpha_{1}+1\right) \ldots\left(2 \alpha_{r}+1\right)+1}{2} .
$$


Demonstração. De acordo com o Lema 2.6, os conjuntos (grupos aditivos abelianos) $A_{i}$ podem ser particionados com relação as suas classes $l^{2}$-ciclotômicas $X_{p_{i}^{\beta_{i}}}$, onde $0 \leq \beta_{i} \leq \alpha_{i}-1 \mathrm{e}$ $1 \leq i \leq r$, além das classes do tipo $C_{0}$ módulo $p_{i}^{\alpha_{i}}$. Ainda, conforme foi afirmado na Observação 2.5, qualquer classe $l$-ciclotômica pode ser vista como $\lambda$-imagem de uma combinação arbitrária de classes $l^{2}$-ciclotômicas. Por hora desconsideremos a classe $C_{0}$ módulo $m$, gerada de forma única pelos zeros dos $A_{i}$, para todo $1 \leq i \leq r$. Logo existem $\left(2 \alpha_{1}+1\right)$ $\left(2 \alpha_{2}+1\right) \ldots\left(2 \alpha_{r}+1\right)-1$ combinações possíveis. Neste cálculo devemos descartar um dos dois tipos de combinações abaixo:

$$
\lambda\left(X_{p_{1}^{\beta_{1}}} \times \ldots \times X_{p_{r}^{\beta_{r}}} \cup Y_{p_{1}^{\beta_{1}}} \times \ldots \times Y_{p_{r}^{\beta_{r}}}\right) \text { ou } \lambda\left(Y_{p_{1}^{\beta_{1}}} \times \ldots \times Y_{p_{r}^{\beta_{r}}} \cup X_{p_{1}^{\beta_{1}}} \times \ldots \times X_{p_{r}^{\beta_{r}}}\right),
$$

pois estas $\lambda$-imagens geram a mesma classe $l$-ciclotômica.

Assim existem $\frac{\left(2 \alpha_{1}+1\right)\left(2 \alpha_{2}+1\right) \ldots\left(2 \alpha_{r}+1\right)-1}{2}$ classes $l$ ciclotômicas. Adicionando mais uma classe, a saber, a classe $C_{0}$ módulo $m$ e a partir de algumas manipulações, verificamos a igualdade

$$
\frac{\left(2 \alpha_{1}+1\right) \ldots\left(2 \alpha_{r}+1\right)+1}{2}=2^{r-1} \prod_{i=1}^{r} \alpha_{i}+2^{r-2} \sum_{\substack{k=1 \\ r}}^{r} \prod_{\substack{i=1 \\ i \neq k}}^{r} \alpha_{i}+\ldots+2 \sum_{1 \leq i<j \leq r} \alpha_{i} \alpha_{j}+\sum_{i=1}^{r} \alpha_{i}+1
$$

Aqui desenvolvemos um método para explicitar os idempotentes primitivos de $\mathcal{R}_{m}$ descrevendo-os como $\lambda$-produto de idempotentes primitivos de $\mathcal{R}_{p_{i} \alpha_{i}}$, para cada $1 \leq i \leq r$.

Definição 2.8. Para $1 \leq i \leq r$, seja $f_{i}(x)=\sum_{j_{i}=0}^{p_{i}^{\alpha_{i}}-1} a_{j_{i}} x^{j_{i}} \in \mathbb{F}_{l^{2}}[x]$. Definimos

$$
\lambda\left(f_{1}(x) \otimes \ldots \otimes f_{r}(x)\right)=\sum_{\lambda\left(j_{1}, \ldots, j_{r}\right) \in \lambda\left(A_{1} \times \ldots \times A_{r}\right)} a_{j_{1}} \cdot \ldots \cdot a_{j_{r}} x^{\lambda\left(j_{1}, \ldots, j_{r}\right)} .
$$

Chamamos este produto de $\lambda$-produto dos polinômios $f_{i}(x)=\sum_{j_{i}=0}^{p_{i}^{\alpha_{i}}-1} a_{j_{i}} x^{j_{i}}$.

\section{Observação 2.9 .}

(i) Para $1 \leq i \leq r$ e $0 \leq \beta_{i} \leq \alpha_{i}-1$, denotemos por $\theta_{b p_{1}^{\beta_{1}} p_{2}^{\beta_{2}} \ldots p_{r}^{\beta_{r}}}$ o idempotente primitivo correspondente a classe $l$-ciclotômica $C_{b p_{1}^{\beta_{1}} p_{2}^{\beta_{2}} \ldots . p_{r}^{\beta_{r}}}$ no anel semissimples $\mathcal{R}_{m}$, onde $l^{t} \not \equiv b \in A, \operatorname{com} t \in \mathbb{N}$ e $m d c\left(b, \prod_{i=1}^{r} p_{i}\right)=1$.

(ii) Denotemos por $\theta_{p_{i}^{\beta_{i}}}^{\beta_{i}}$ e $\theta_{p_{i}^{\beta_{i}}}^{*_{*}}$ os idempotentes primitivos básicos correspondentes as classes $l^{2}$-ciclotômicas $X_{p_{i}^{\beta_{i}}}$ e $Y_{p_{i}^{\beta_{i}}}$, respectivamente no anel $\mathcal{R}_{p_{i}^{\alpha_{i}}}$. Além disso, dado $s \in A$, defina $\sigma_{s}(x)=\sigma_{C_{s}}(x)=\sum_{t \in C_{s}} x^{t}$.

(iii) Sejam $X_{1} \times \ldots \times X_{r}, X_{p_{1}^{\beta_{1}}} \times \ldots \times X_{p_{r}^{\beta_{r}}}$ e $\theta_{p_{1}^{\beta_{1}}}^{*} \otimes \ldots \otimes \theta_{p_{r}^{\beta_{r}}}^{*}$ as primeiras partes das expressões $\lambda\left(X_{1} \times \ldots \times X_{r} \cup Y_{1} \times \ldots \times Y_{r}\right), \quad \lambda\left(X_{p_{1}^{\beta_{1}}} \times \ldots \times X_{p_{r}^{\beta_{r}}} \cup Y_{p_{1}^{\beta_{1}}} \times \ldots \times Y_{p_{r}^{\beta_{r}}}\right) \quad \mathrm{e}$ $\lambda\left(\theta_{p_{1}^{\beta_{1}}}^{*} \otimes \ldots \otimes \theta_{p_{r}^{\beta_{r}}}^{*}\right)+\lambda\left(\begin{array}{c}\theta_{p_{1}^{\beta_{1}}}^{* *} \otimes \ldots \otimes \theta_{p_{r}^{\beta_{r}}}^{* *} \\ { }^{*}\end{array}\right)$, respectivamente. Ainda, $X_{i}, X_{p_{i}^{\beta_{i}}}$ e $\theta_{p_{i}^{\beta_{i}}}^{*}$ são as $i$-ésimas entradas de $X_{1} \times \ldots \times X_{r}, X_{p_{1}^{\beta_{1}}} \times \ldots \times X_{p_{r}^{\beta_{r}}}$ e $\theta_{p_{1}^{\beta_{1}}}^{*} \otimes \ldots \otimes \theta_{p_{r}^{\beta_{r}}}^{*}$, respectivamente. 
Teorema 2.10. Se, para cada $0 \leq \beta_{i} \leq \alpha_{i}$, as i-ésimas entradas da primeira parte de

$$
\begin{gathered}
C_{1}=\lambda\left(X_{1} \times \ldots \times X_{r} \cup Y_{1} \times \ldots \times Y_{r}\right) e \\
C_{b p_{1}^{\beta_{1}} p_{2}^{\beta_{2}} \ldots . p_{r}^{\beta_{r}}}=\lambda\left(X_{p_{1}^{\beta_{1}}} \times \ldots \times X_{p_{r}^{\beta_{r}}} \cup Y_{p_{1}^{\beta_{1}}} \times \ldots \times Y_{p_{r}^{\beta_{r}}}\right),
\end{gathered}
$$

são $R_{i}$ e $R_{p_{i}^{\beta_{i}}}\left(\right.$ ou $\left.N_{p_{i}^{\beta_{i}}}\right)$, então $\theta_{b p_{1}^{\beta_{1}} p_{2}^{\beta_{2}} \ldots \cdot p_{r}^{\beta_{r}}}=\lambda\left(\theta_{p_{1}^{\beta_{1}}}^{*} \otimes \ldots \otimes \theta_{p_{r}^{\beta_{r}}}^{*}\right)+\lambda\left(\begin{array}{c}\theta_{p_{1}^{\beta_{1}}}^{* *} \otimes \ldots \otimes \theta_{p_{r}^{\beta_{r}}}^{* *} \\ { }^{*}\end{array}\right)$, onde, para $1 \leq i \leq r, \underset{p_{i}^{\beta_{i}}}{*^{*}} \theta_{p_{i}^{\beta_{i}}}^{* *}$ são os idempotentes primitivos correspondentes as classes $l^{2}$-ciclotômicas $R_{p_{i}^{\beta_{i}}}\left(\right.$ ou $\left.N_{p_{i}^{\beta_{i}}}\right)$ e $N_{p_{i}^{\beta_{i}}}\left(\right.$ ou $\left.R_{p_{i}^{\beta_{i}}}\right)$, respectivamente.

\section{Segunda abordagem - álgebra de grupo}

\subsection{Códigos Abelianos Minimais}

Sejam $\mathbb{F}_{2}$ o corpo finito com 2 elementos e $G$ um grupo finito de ordem ímpar. Um ideal minimal de $\mathbb{F}_{2} G$ será dito um código abeliano binário minimal. Nesta seção apresentamos as expressões dos idempotentes primitivos geradores dos códigos binários cíclicos de comprimento $p^{m} q^{n}, p q$ e $p_{1} p_{2} p_{3}$, onde $p, q, p_{1}, p_{2}$ e $p_{3}$ são todos primos ímpares distintos dois a dois. Iniciamos com dois resultados técnicos. Em [5], foram determinados todos os códigos minimais sob as seguintes hipóteses:

$G$ é um grupo abeliano finito de expoente $p^{m}$ ou $\left(2 p^{m}\right)$,

$\mathbb{F}$ um corpo finito com q elementos onde $q$ tem ordem multiplicativa $\varphi\left(p^{m}\right) \bmod p^{m}$.

Em [2], os autores fizeram o estudo dos idempotentes primitivos para grupos da forma $G_{p} \times G_{q}$, onde $G_{p}$ e $G_{q}$ denotam grupos abelianos, o primeiro um $p$-grupo e o segundo um $q$-grupo, que satisfazem as seguintes condições, que, em particular, satisfazem (10):

$$
\begin{aligned}
& m d c(p-1, q-1)=2, \\
& \overline{2} \text { gera o grupo das unidades de } \mathbb{Z}_{p^{2}} \text { e } \mathbb{Z}_{q^{2}} \\
& m d c(p-1, q)=m d c(p, q-1)=1 .
\end{aligned}
$$

Observação 3.1. Em (11), a primeira hipótese nos garante que pelo menos um dos números primos $p$ e q é da forma $4 k+3$, onde $k \in \mathbb{N}$.

Seja $G$ um grupo de ordem ímpar. Para um subgrupo $H \leq G$, definimos $\widehat{H}=\frac{1}{|H|} \sum_{h \in H} h$, da mesma forma que [5] e, para um elemento $x \in G, \widehat{x}=\widehat{\langle x\rangle}$.

\subsection{Códigos em $\mathbb{F}_{2}\left(\mathcal{C}_{p} \times \mathcal{C}_{q}\right)$}

Sejam $p \neq q$ primos ímpares. Considere o grupo $G=\left\langle g \mid g^{p q}=1\right\rangle, a=g^{q}, b=g^{p}$ e $G=\mathcal{C}_{p} \times \mathcal{C}_{q}$, onde $\mathcal{C}_{p}=\langle a\rangle$ e $\mathcal{C}_{q}=\langle b\rangle$. Nesta seção, descrevemos todos os idempotentes primitivos de $\mathbb{F}_{2}\left(\mathcal{C}_{p} \times \mathcal{C}_{q}\right)$.

Teorema 3.2 ( [2], Theorem IV.1). Seja $G=\langle a\rangle \times\langle b\rangle$ como acima e assuma que $p$ e $q$ satisfazem (11). Então os idempotentes primitivos de $\mathbb{F}_{2} G$ são

$$
e_{0}=\widehat{G}, \quad e_{1}=\widehat{a}(1-\widehat{b}), \quad e_{2}=(1-\widehat{a}) \widehat{b}, \quad e_{3}=u v+u^{2} v^{2} \text { e } e_{4}=u v^{2}+u^{2} v \text {, }
$$

onde,

$$
u(a)= \begin{cases}a^{2^{0}}+a^{2^{2}}+\cdots+a^{2^{p-3}}, & \text { se } p \equiv 1(\bmod 4) \text { ou } \\ 1+a^{2^{0}}+a^{2^{2}}+\cdots+a^{2^{p-3}}, & \text { se } p \equiv 3(\bmod 4)\end{cases}
$$

e

$$
u^{\prime}(a)= \begin{cases}a^{2}+a^{2^{3}}+\cdots+a^{2^{p-2}}, & \text { se } p \equiv 1(\bmod 4) \text { ou } \\ 1+a^{2}+a^{2^{3}}+\cdots+a^{2^{p-2}}, & \text { se } p \equiv 3(\bmod 4)\end{cases}
$$


3.3 Códigos em $\mathbb{F}_{2}\left(\mathcal{C}_{p^{m}} \times \mathcal{C}_{q^{n}}\right)$, para $m \geq 2, n \geq 2$

Teorema 3.3 ( [2], Theorem V.1). Sejam $p$ e q números primos que satisfazem (11) e $G=$ $\langle a\rangle \times\langle b\rangle$, com $\langle a\rangle=\mathcal{C}_{p^{m}}$ e $\langle b\rangle=\mathcal{C}_{q^{n}}$. Então os códigos minimais de $\mathbb{F}_{2} G$ são gerados pelos seguintes idempotentes primitivos

$$
e_{0}=\widehat{a} \widehat{b}, \quad e_{0 j}=\widehat{a}\left(\widehat{b^{q^{j}}}+\widehat{b^{q^{j-1}}}\right), \quad e_{i 0}=\left(\widehat{a^{p^{i}}}+\widehat{a^{p^{i-1}}}\right) \widehat{b}, \quad e_{i j}^{*}=u v+u^{2} v^{2} \text { e } e_{i j}^{* *}=u v^{2}+u^{2} v,
$$

onde

$$
\begin{aligned}
& u=\widehat{a^{p^{i}}}\left(a^{2^{0} p^{i-1}}+a^{2^{2} p^{i-1}}+\cdots+a^{2^{p-3} p^{i-1}}\right), \text { se } p \equiv 1(\bmod 4) \text { ou } \\
& u=\widehat{a^{p^{i}}}\left(1+a^{2^{0} p^{i-1}}+a^{2^{2} p^{i-1}}+\cdots+a^{2^{p-3} p^{i-1}}\right), \text { se } p \equiv 3(\bmod 4)
\end{aligned}
$$

$e$

$$
\begin{aligned}
& v=\widehat{b^{j}}\left(b^{2^{0} q^{j-1}}+b^{2^{2} q^{j-1}}+\cdots+b^{2^{q-3} q^{j-1}}\right), \text { se } q \equiv 1(\bmod 4) \text { ou } \\
& v=\widehat{b^{q^{j}}}\left(1+b^{2^{0} q^{j-1}}+b^{2^{2} q^{j-1}}+\cdots+b^{2^{q-3} q^{j-1}}\right), \text { se } q \equiv 3(\bmod 4)
\end{aligned}
$$

\subsection{Códigos em $\mathbb{F}_{2}\left(\mathcal{C}_{p_{1}} \times \mathcal{C}_{p_{2}} \times \mathcal{C}_{p_{3}}\right)$}

Os métodos das seções anteriores podem ser estendidos para o caso geral, mas os cálculos tornamse muito mais complexos. Como uma ilustração, mostramos abaixo como obter os idempotentes primitivos quando $|G|$ envolve três primos distintos.

Teorema 3.4 ( [2], Theorem IV.10). Sejam $p_{1}, p_{2}$ e $p_{3}$ três primos impares distintos tais que $m d c\left(p_{i}-1, p_{j}-1\right)=2$, para $1 \leq i \neq j \leq 3$, e $\overline{2}$ um gerador do grupo $U\left(\mathbb{Z}_{p_{i}}\right)$. Então os idempotentes primitivos da álgebra de grupo $\mathbb{F}_{2} G$, para $G=\mathcal{C}_{p_{1}} \times \mathcal{C}_{p_{2}} \times \mathcal{C}_{p_{3}}$, com $\mathcal{C}_{p_{1}}=\langle a\rangle$, $\mathcal{C}_{p_{2}}=\langle b\rangle$ e $\mathcal{C}_{p_{2}}=\langle c\rangle$, são

$$
\begin{array}{ll}
e_{0}=\hat{a} \hat{b} \hat{c} & e_{1}=\hat{a} \hat{b}(1-\hat{c}) \\
e_{2}=\hat{a}(1-\hat{b}) \hat{c} & e_{3}=(1-\hat{a}) \hat{b} \hat{c} \\
e_{4}=\left(u v+u^{2} v^{2}\right) \hat{c} & e_{5}=\left(u^{2} v+u v^{2}\right) \hat{c} \\
e_{6}=\left(u w+u^{2} w^{2}\right) \hat{b} & e_{7}=\left(u^{2} w+u w^{2}\right) \hat{b} \\
e_{8}=\left(v w+v^{2} w^{2}\right) \hat{a} & e_{9}=\left(v^{2} w+v w^{2}\right) \hat{a} \\
e_{10}=(1-\hat{a})(1-\hat{b})(1-\hat{c})+u^{2} v^{2} w+u v w^{2} & e_{11}=(1-\hat{a})(1-\hat{b})(1-\hat{c})+u^{2} v^{2} w^{2}+u v w \\
e_{12}=(1-\hat{a})(1-\hat{b})(1-\hat{c})+u^{2} v w^{2}+u v^{2} w^{2} & e_{13}=(1-\hat{a})(1-\hat{b})(1-\hat{c})+u v^{2} w+u^{2} v w^{2}
\end{array}
$$

onde $u=u(a), v=v(b)$ e $w=w(c)$ são definidos como em 3.2, respectivamente.

\section{Análise das técnicas apresentadas}

Ambas as técnicas apresentadas neste trabalho nos permitem calcular os idempotentes primitivos dos códigos cíclicos minimais de modo algorítmico. A partir do cálculo de exemplos específicos, observamos que as fórmulas apresentadas em [6] podem conter alguns erros em seus coeficientes. A limitação de espaço nos fez optar por não apresentar o exemplo neste resumo.

As técnicas polinomiais envolvem a utilização de extensões do corpo base e, algumas vezes, descuidam do fato de que o produto de idempotentes primitivos nem sempre é primitivo.

Por outro lado, a técnica de álgebra de grupo se mostrou muito eficiente no cálculo dos idempotentes primitivos, utilizando simplesmente a estrutura dos subgrupos do grupo subjacente, o que evita, por exemplo, o uso de raízes primitivas, usadas na técnica polinomial. Outra vantagem é que fica muito claro de onde se origina cada idempotente primitivo a partir da estrutura do grupo. Isto facilita, por exemplo, determinar certas equivalências de códigos minimais, 
conforme [4], e também nos permite determinar claramente quantos fatores primitivos aparecem quando o produto de dois idempotentes primitivos não é primitivo.

Pelos estudos que realizamos até o momento dos desenvolvimentos dessas técnicas, acreditamos que uma boa combinação de ambas possa minimizar possíveis erros nas expressões dos idempotentes.

\section{Referências}

[1] S.K. Arora and M. Pruthi Minimal cyclic codes of length $2 p^{n}$. Finite Fields Appl. 5 no. 2 (1999) $177-187$.

[2] G. Chalom, R. A. Ferraz, M. Guerreiro and C. Polcino Milies Minimal Binary Abelian Codes of length $p^{m} q^{n}$. preprint in arXiv:1205.5699.

[3] R. Ferraz Simple components and central units in group algebras. J. Algebra, 279 (2004) 191-203.

[4] R. Ferraz, M. Guerreiro, C. Polcino Milies, G-equivalence in group algebras and minimal abelian codes, IEEE Transactions on Information Theory, 60 N. 1 (2014) 252-260.

[5] R. Ferraz, C. Polcino Milies, Idempotents in group algebras and minimal abelian codes, Finite Fields and their Appl., 13, (2007) 382-393.

[6] P. Kumar e S.K. Arora $\lambda$-Mapping and Primitive Idempotents in semi simple ring $R_{m}$. Communications in Algebra, to appear

[7] I. Niven, H.S. Zuckerman, H.L. Montgomery, An Introduction to the Theory of Numbers, 5th Edition, John Wiley, New York, 1991.

[8] C. Polcino Milies, S.K. Sehgal, An Introduction to Group Rings, Kluwer Academic Publishers, Dordrecht, 2002.

[9] M. Pruthi and S.K. Arora Minimal cyclic codes of prime power length. Finite Fields Appl. 3 no. 2 (1997) 99-113. 